\title{
SPECTRUM LINES AS LIGHT SOURCES IN POLARISCOPIC MEASUREMENTS.
}

\author{
By Frederick Bates.
}

Recent progress in polariscopic work necessitates careful consideration of the character and behavior of the light sources used. Unless the source can be accurately defined and its constancy depended upon, it is obviously impossible to compare the polariscopic measurements of different observers.

In the half-shade polariscope the circular field is divided into two equal parts by a black line. When a setting of the instrument is

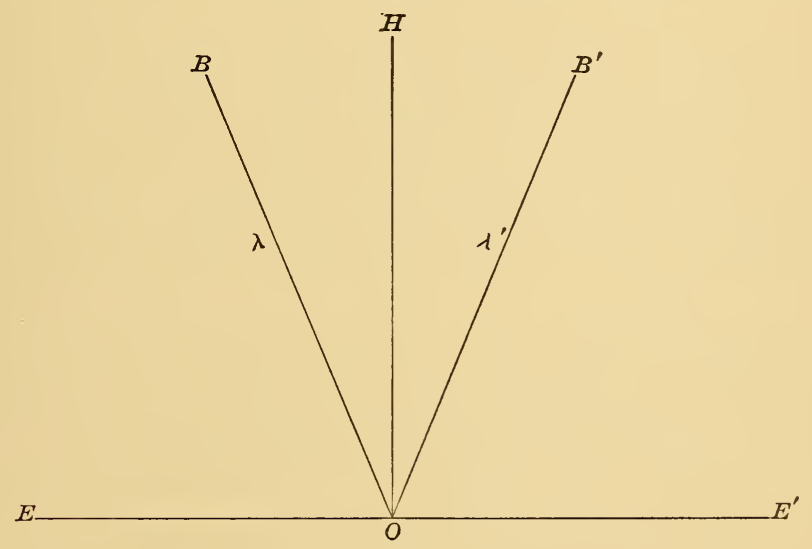

Fig. 1.

made the analyzer is rotated into a position which makes the illumination of the two halves equal. This condition is represented in Fig. I.

The normal $\mathrm{HO}$ to the polarization plane $\left(\mathrm{EE}^{\prime}\right)$ of the analyzer bisects the angle $\mathrm{BOB}^{\prime}$ where $\mathrm{BO}$ and $\mathrm{B}^{\prime} \mathrm{O}$ are the traces of the polarization planes of the large and small nicols of the polarizing 
system. If the source be monochromatic and a rotation takes place anywhere between the polarizing system and the analyzer, $\mathrm{HO}$ again bisects $\mathrm{BOB}^{\prime}$ when the condition of equal illumination is established. If the source be not monochromatic, only the polarization planes of the waves with the same wave length coincide after the rotation occurs.

Let a source, as shown in Fig. 2, consist of two different wave lengths, one of which is $\lambda_{1}$ and $\lambda_{1}^{\prime}$ in the left and right halves of

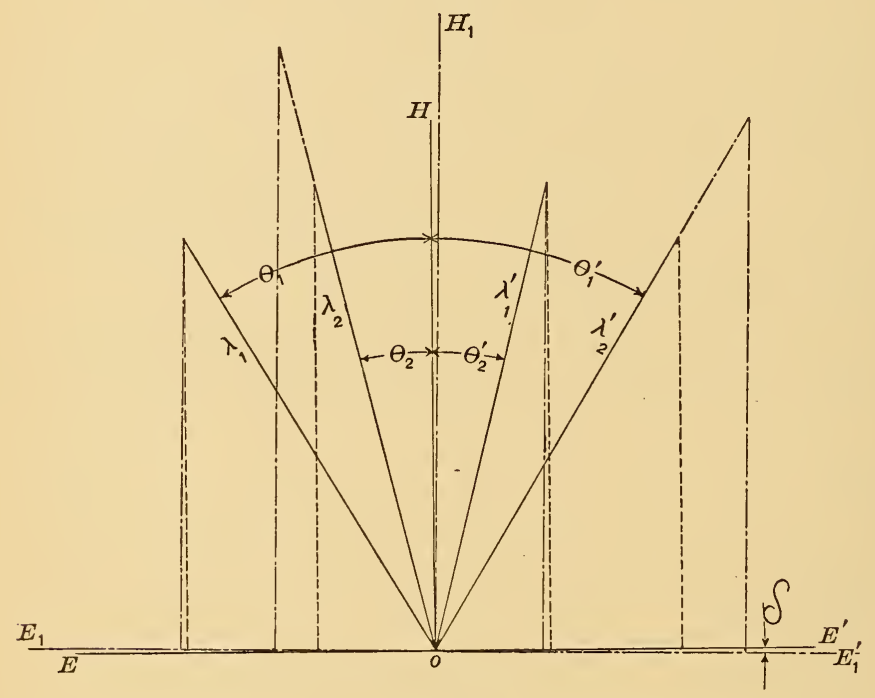

Fig. 2.

the field respectively; and the other similarly $\lambda_{2}$ and $\lambda_{2}^{\prime}$. Let the intensities of $\lambda_{1}$ and $\lambda_{1}^{\prime}$ be $A_{1}$, and of $\lambda_{2}$ and $\lambda^{\prime}{ }_{2}$ be $A_{2}$. When $E E$ occupies the position for equal illumination we have

$$
A_{1} \sin { }^{2} \theta_{1}+A_{2} \sin { }^{2} \theta_{2}=A_{1} \sin { }^{2} \theta^{\prime}{ }_{2}+A_{2} \sin { }^{2} \theta^{\prime}{ }_{1}
$$

in which the right and left hand members are the intensities of the corresponding halves of the field as seen through the analyzer.

Let

$$
\begin{array}{rlrl}
\theta_{1} & =\phi_{1} \pm \delta & \theta_{2} & =\phi_{2} \pm \delta \\
\theta^{\prime}{ }_{1}=\phi_{1} \mp \delta & \theta^{\prime}{ }_{2}=\phi_{2} \mp \delta
\end{array}
$$

Equation (I) becomes

$$
A_{1} \sin ^{2}\left(\phi_{1} \pm \delta\right)+A_{2} \sin ^{2}\left(\phi_{2} \pm \delta\right)=A_{1} \sin ^{2}\left(\phi_{2} \mp \delta\right)+A_{2} \sin ^{2}\left(\phi_{1} \mp \delta\right)(2)
$$


Solving (2) for $\delta$ by substituting

$$
\frac{A_{2}}{A_{1}}=K \text { and } \sin ^{2} \theta=\frac{1}{2}(\mathrm{I}-\cos 2 \theta)
$$

we obtain

$$
\tan 2 \delta= \pm \frac{K-\mathrm{I}}{K+\mathrm{I}} \tan \left(\phi_{1}-\phi_{2}\right)
$$

or since all the angles are small

$$
\delta= \pm \frac{1}{2} \frac{K-\mathrm{I}}{K+\mathrm{I}}\left(\phi_{1}-\phi_{2}\right)
$$

When $\mathrm{K}=\mathrm{I}$ we have

$$
\delta=0 ; \theta_{1}=\theta^{\prime}{ }_{1} ; \theta_{2}=\theta^{\prime}{ }_{2}
$$

Equation (2) then expresses the condition for equal illumination when measuring a rotation for a wavelength which is the so-called optical center of gravity of the two wavelengths when they have equal amplitudes.

Only in rare instances is $\mathrm{K}$ unity. Thus $\delta$ is a rotation which is the difference between the rotation when the intensities corresponding to the two wavelengths are equal, and the rotation when they are unequal. In general, when the shorter of the two wavelengths has the greater intensity the sign of $\delta$ is positive, and it is in the same direction as the rotation of the substance. By means of (4) we easily calculate the change in the rotation for any variation in the intensity of either wave. With a given line structure and $\mathrm{K}$ constant, $\delta$ is a function of $\left(\phi_{1}-\phi_{2}\right)$ only, which in turn is a function of the rotation dispersion curve. With certain substances showing anomalous rotation dispersion the sign of $\left(\phi_{1}-\phi_{2}\right)$ might be reversed. Hence the assumption which has long been accepted as a fact ${ }^{1}$ that the optical center of gravity is independent of the rotation dispersion is incorrect.

The angular rotation $\delta$ resulting from increasing the intensity of one of the waves is shown graphically in Fig. 2. 
In the case of the two spectral lines of incandescent sodium vapor used as a source, for a thickness of quartz giving a rotation of about $253^{\circ}$

$$
\begin{array}{rlrl}
\lambda_{1} & =589.62 & A_{1} & =\mathrm{I} \\
\lambda_{2} & =5^{89.02} & A_{2} & =2 \\
\left(\phi_{1}-\phi_{2}\right) & =0.466^{\circ} & & \\
\operatorname{in}(4) & & K=\mathrm{I} .6 . \\
\delta & =\frac{\mathrm{r} .6-\mathrm{I}}{2(\mathrm{I} .6+\mathrm{I})} \mathrm{O} .466 & =0.054^{\circ} .
\end{array}
$$

Substituting in (4)

The total rotation measured is therefore $0.054^{\circ}$ greater than that which would have been obtained with $\lambda=589.32 \mu \mu$ (mean of $\lambda_{1}+\lambda_{2}$ ), which gives $589.25 \mu \mu$ as the optical center of gravity of these lines for all substances having approximately the same rotation dispersion as quartz.

Owing to the fact that they are easily obtained quite intense and free from the presence of other lines, the two spectral sodium lines $\lambda=5^{89} .62$ and 589.02 have been very extensively used as a monochromatic source. All of the optical constants used in polariscopic work have been determined with them. After an extensive investigation the writer has found no sodium source that is an improvement on sticks of fused $\mathrm{Na}_{2} \mathrm{CO}_{3}$ fed into an oxhydrogen flame. However, the lack of intensity and unstable line structure under certain conditions renders even this source far from satisfactory, owing to the great precision required in polariscopic measurements. In utilizing this source the rod of fused $\mathrm{Na}_{2} \mathrm{CO}_{3}$ is placed in the flame at one of the positions shown in Fig. 3.

Through the kindness of Dr. P. G. Nutting it was possible to examine the line structure of the sodium lines as well as that of the line $\lambda=546$. I $\mu \mu$ of mercury vapor with an echelon spectroscope. Table I refers to Fig. 3.

The lines obtained by this method are extremely sharp and differ from the arc spectra by the absence of the characteristic haziness of the edges. If displacements as large as $0.5 \mu \mu$ had occurred, they would have been readily detected. The broadening was at all times symmetrical. In this respect the observations agree with the more recent work of other observers, but are contradictory to the results obtained by Ebert ${ }^{2}$ which have been accepted in polariscopic work. 
By careful manipulation of the flame in position 3 reversal of the lines took place. $\mathrm{D}_{2}$ preceded $\mathrm{D}_{1}$ in this respect.

It is thus evident that so far as their line structure is concerned

TABLE I.

Structure of Sodium Lines at Different Intensities.

\begin{tabular}{|c|c|c|c|c|c|c|}
\hline & \multirow[b]{2}{*}{$\begin{array}{r}\text { Relative } \\
\text { Intensity }\end{array}$} & \multirow[b]{2}{*}{$\begin{array}{l}\text { Position I, } \\
\text { Width } \mu \mu\end{array}$} & \multirow[b]{2}{*}{$\begin{array}{l}\text { Position 2, } \\
\text { Width } \mu \mu\end{array}$} & \multicolumn{3}{|c|}{ Position 3 , Width $\mu \mu$} \\
\hline & & & & $\begin{array}{l}\mathrm{Na}_{2} \mathrm{CO}_{3} \mathrm{Melt}- \\
\text { ing Slowly }\end{array}$ & $\mathrm{Na}_{2} \mathrm{CO}_{3} \mathrm{I}$ & $\begin{array}{l}\text { ting Very } \\
\text { ly }\end{array}$ \\
\hline$D_{x}$ & 1 & 0.008 & 0.02 & 0.3 to 0.4 & $\begin{array}{c}0.4 \\
\text { Reversed }\end{array}$ & $\begin{array}{c}\text { Reversed } \\
0.6 \\
\text { Reversed }\end{array}$ \\
\hline$D_{2}$ & 1.6 & 0.008 & 0.02 & 0.3 to 0.4 & 0.6 & 0.6 \\
\hline
\end{tabular}

the sodium lines can be depended upon to give a sufficiently definite optical center of gravity up to the point of reversal in position 3 . However, very noticeable variations in polariscopic measurements are likely to be observed with sodium sources at different intensities. These variations it is believed are not due to changes in the line structure of the source, but to the difficulty of excluding all extraneous light even with a very narrow slit and a dispersion sufficient to separate $\mathrm{D}_{1}$ and $\mathrm{D}_{2}$. This extraneous light constitutes a different percentage of the total illumination whenever the intensity of the source varies. It may, therefore, appreciably affect the optical center of gravity when the field appears dim, and inversely when bright. Aside from the color and stability of the sodium lines up to reversal there is nothing in their favor as a polariscopic source. The flame requires the

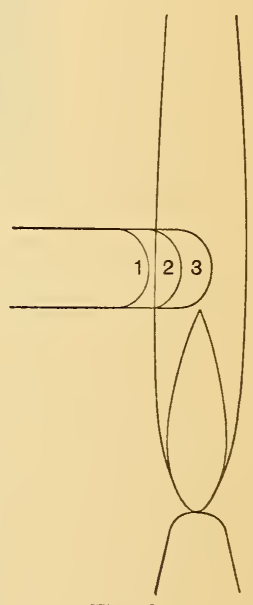

Fig. 3. constant attention of an assistant, and even in reversal the intensity is not nearly sufficient to permit of the use of the greatest sensibility of a good polarizing system.

It is proposed to use the green line of incandescent mercury vapor $\lambda=546$. I $\mu \mu$ as the standard source for all accurate polariscopic work. Quartz mercury-vapor lamps as now made are reliable in 
action. If sufficient care is exercised in preparing the mercury and exhausting the lamps, the five characteristic lines only will be obtained in the visible spectrum and with great intensity.

Different observers have found markedly different line structures for the line $\lambda=546$. I $\mu \mu$ depending upon the method of analysis employed. The map in Fig. 4 was obtained with the echelon with I. 8 amperes passing through the lamp. The fractional values given are the relative intensities as nearly as they could be estimated. It was found impossible to decide whether the satellite -.024 belongs to the positive or negative side of the primary. When the current was increased to more than 2.I amperes the satellite -.055 increasd in intensity until it about equaled the primary. The difference in wave lengths of the extreme satellites is less than $0.04 \mu \mu$. With a

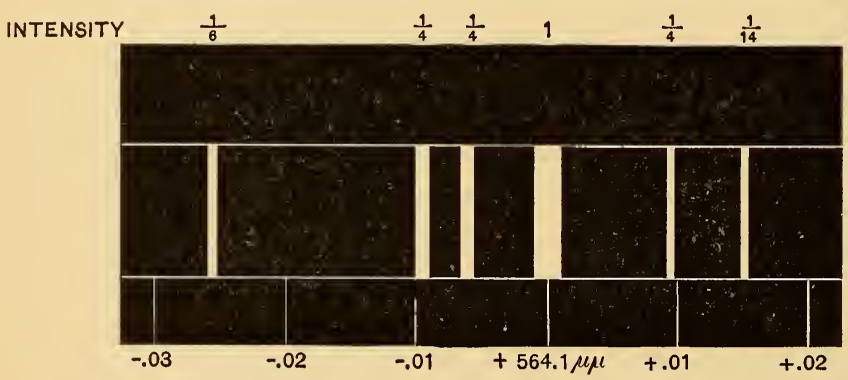

Fig. 4.

different source Fabry and Perot $^{3}$ found $0.035 \mu \mu$ and Houstoun $0.02 \mathrm{I} 5 \mu \mu$. The distance between $\mathrm{D}_{1}$ and $\mathrm{D}_{2}$ is fifteen times $0.04 \mu \mu$. As far as it has been possible to determine, the line structure for the quartz lamp under widely varying conditions is such that for polariscopic purposes $\lambda=546$. I $\mu \mu$ is a monochromatic source of great intensity and perfect reliability. In measuring a rotation of two hundred and fifty degrees no differences could be detected due to changes in the emission, and probably none for much larger rotations. The quartz lamp requires little attention and can be operated indefinitely. Since only the lines $\lambda=579.0,576.9,546.1,435.8$, and 404.7 $\mu \mu$ are present the difference in wave length is such as to permit of perfect separation of the line 546.I $\mu \mu$ by even a relatively

${ }^{3}$ C. R., I898, p. 409.

${ }^{4}$ Houstoun Phil. Mag. 7, p. 456 (1904). 
small dispersion, and without bringing other lines in close proximity to the edges of the slit. Hence this source permits the elimination of practically all diffused light.

546. I $\mu \mu$ by even a relatively small dispersion, and without bringing other lines in close proximity to the edges of the slit. Hence this source permits the elimination of practically all diffused light.

In order to make the advantages of the above system available, the temperature coefficient ( $a$ ) of quartz for $\lambda=546$.I $\mu \mu$ as well as the constant $\frac{\phi_{\lambda=546.1}}{\phi_{\lambda=589.2}}$ (where $\phi$ is a rotation) were determined. It was thought advisable to measure $a$, although V. Lang, ${ }^{5}$ Sohncke, ${ }^{6}$ and Le Chatelier ${ }^{7}$ state that it has the same value for all wave lengths.

We have

$$
\phi_{\mathrm{t}}=\phi_{\mathrm{o}}(\mathrm{I}+a t)
$$

where $\phi_{\mathrm{t}}$ is the rotation at temperature $t$ and $\phi_{\mathrm{o}}$ at zero. For any other temperature $t$ we have

$$
\begin{gathered}
\phi_{\mathrm{t}_{1}}=\phi_{\mathrm{o}}\left(\mathrm{I}+a t_{1}\right) \\
a=\frac{\phi_{\mathrm{t}_{1}}-\phi_{\mathrm{t}}}{\phi_{\mathrm{t}} t_{1}-\phi_{\mathrm{t}_{1}} t}
\end{gathered}
$$

Hence

The measurements were made with the most improved types of apparatus. $\lambda=546$.I $\mu \mu$ was separated by means of two dense prisms. The left rotating quartz plate was placed in a water cell closed with glass end plates. The cell could be opened and the quartz removed without disturbing the mounting of these plates. This is of the greatest importance, as it insures constancy of the optical path at all times save that caused by the presence and absence of the quartz plate. Water at the desired temperature flowed through the glass cell during all measurements. Twenty consecutive settings were always made-five on the zero point, ten with the quartz inserted, and five on the zero. A summary of the data is given in Table II.

By combining the values of $\phi$ at the different temperatures from the data in Table II we obtain the following:

$$
a=0.000144 \pm 0.00000087
$$

${ }^{5}$ V. Lang: Pogg. Annalen 156,422 (1875).

${ }^{6}$ Sohncke: Wied. Annalen 3,516.

${ }^{7}$ Le Chatelier: C. R. Io9-244 (1889). 
Hence

$$
\phi_{\mathrm{t}}=\phi_{\mathrm{o}}(\mathrm{I}+0.000 \mathrm{I} 44 t)
$$

between $4^{\circ} \mathrm{C}$ and $50^{\circ} \mathrm{C}$.

In measuring $\frac{\phi_{\lambda=546.1}}{\phi_{\lambda=589.25}}$ a very large number of determinations were

TABLE II.

Rotation for $\lambda=546.1 \mu \mu$.

\begin{tabular}{|c|c|c|}
\hline $\begin{array}{l}\text { Observa- } \\
\text { tion No. }\end{array}$ & Temperature & Rotation $\phi$ \\
\hline 1 & $4: 854 \mathrm{C}$ & $136: 8968$ \\
\hline 2 & $6: 230$ & $136: 9258$ \\
\hline 3 & $8: 302$ & $136: 9714$ \\
\hline 4 & $16: 049$ & $137: 0984$ \\
\hline 5 & $20: 761$ & $137: 1958$ \\
\hline 6 & $29: 575$ & $137: 3853$ \\
\hline 7 & $44: 568$ & 137.6832 \\
\hline 8 & $49: 799$ & $137: 7929$ \\
\hline
\end{tabular}

TABLE III.

Value of $\phi$.

\begin{tabular}{|c|c|}
\hline $\begin{array}{l}\text { Observations } \\
\text { used. Nos. }\end{array}$ & $a$ \\
\hline 1 and 5 & 0.0001377 \\
\hline 2166 & 0.0001439 \\
\hline 3 " 7 & 0.0001436 \\
\hline 4 " 8 & 0.0001505 \\
\hline 1 " 7 & 0.0001447 \\
\hline 1 " 8 & 0.0001481 \\
\hline 2 " 7 & 0.0001444 \\
\hline 2 " 68 & 0.0001430 \\
\hline
\end{tabular}

made, practically all of which were concordant. However, in order to eliminate the personal equation and avoid as far as possible errors due to the character of the sodium source, the value of $\phi$ is computed from the measurements of five plates whose sodium value has recently been determined at the Physikalisch-Technische 
Reichsanstalt. The mean of this value and that of the writer was taken as the rotation for $\lambda=589.25$. The rotations were measured in part with a sensitive strip polarizing system. The greater number, however, were made with an exceptionally good Lippich system. The room temperature was held near to $20^{\circ} \mathrm{C}$. The largest probable error given by the Physikalisch-Technische Reichsanstalt was $\pm .0006^{\circ}$; by the writer, $\pm .0004^{\circ}$. A set of readings for $\lambda=546$. I $\mu \mu$ on Plate No. 4, selected at random, is given in Table IV.

TABLE IV.

Readings on Quartz Plate No. 4.

Plate No. 4. Source $\lambda=546.1 \mu \mu$.

\begin{tabular}{|c|c|c|c|c|}
\hline \multicolumn{2}{|c|}{ Vernier A } & \multicolumn{2}{|c|}{ Vernier B } & \multirow{2}{*}{ T. Deg. } \\
\hline Plate in & Plate out & Plate in & Plate out & \\
\hline $208: 888$ & $176: 364$ & $28: 893$ & $356: 366$ & $20: 90 \mathrm{C}$. \\
\hline $208: 890$ & $176: 366$ & $28: 895$ & $356: 368$ & \\
\hline $208: 889$ & 176.358 & $28: 893$ & $356: 359$ & \\
\hline $208^{\circ} 885$ & $176: 361$ & $28: 890$ & $356: 363$ & \\
\hline $208: 890$ & $176: 364$ & $28: 895$ & $356: 366$ & \\
\hline $208: 889$ & $176: 360$ & $28: 894$ & $356: 362$ & \\
\hline $208: 885$ & 176.358 & $28: 890$ & $356: 361$ & \\
\hline $208: 885$ & $176: 364$ & $28: 890$ & $356: 366$ & \\
\hline $208^{\circ} 885$ & $176: 367$ & $28: 890$ & 356.370 & \\
\hline 208.888 & $176: 362$ & $28: 892$ & $356: 365$ & $20: 80$ \\
\hline
\end{tabular}

From the above

$$
\phi_{\lambda=546 . \mathrm{I}}=32^{\circ} \cdot 522 \text { at } 20^{\circ} \mathrm{C} \text {. }
$$

The data is summarized in Table V, wherein N. B. S. stands for National Bureau of Standards, and P. T. R. for Physikalisch-Technische Reichsanstalt.

We have therefore

$$
\begin{aligned}
& \frac{\phi_{\lambda=546.1}}{\phi_{\lambda=589.25}}=\mathrm{I} . \mathrm{I} 75 \mathrm{I} 66 \pm 0.0000 \mathrm{I} 2 \\
& \frac{\phi_{\lambda=589.25}}{\phi_{\lambda=546.1}}=0.850944
\end{aligned}
$$


TABLE V.

$$
\text { Measurement of } \frac{\phi_{\lambda=546 . I}}{\phi_{\lambda=589.25}}
$$

\begin{tabular}{|c|c|c|c|c|c|}
\hline \multirow{2}{*}{$\begin{array}{l}\text { Plate } \\
\text { No. }\end{array}$} & \multicolumn{3}{|c|}{$\phi_{\lambda}=589.25 ; \mathfrak{t}=20^{\circ} \mathrm{C}$} & \multirow{2}{*}{$\frac{\begin{array}{c}\phi_{\lambda=546.1 ;} ; t= \\
20^{\circ} \mathrm{C}\end{array}}{\text { N. B. S. }}$} & \multirow{2}{*}{$\begin{array}{l}\phi_{\lambda=546.1} \\
\phi_{\lambda=589.25}\end{array}$} \\
\hline & N. B. S. & P. T. R. & Mean & & \\
\hline 1 & 34.5978 & 34.596 & 34.5969 & 40.6581 & 1.17519 \\
\hline 2 & 27.6547 & 27.644 & 27.6493 & 32.4912 & 1.17512 \\
\hline 3 & 34.6292 & 34.626 & 34.6276 & 40.6930 & 1.17516 \\
\hline 4 & 27.672 & 27.676 & 27.674 & 32.5220 & 1.17518 \\
\hline 5 & 20.9169 & 20.916 & 20.9165 & 24.5806 & 1.17518 \\
\hline
\end{tabular}

Thus any quartz rotation for the wave length $589.25 \mu \mu$ may be obtained by measuring the rotation for the wave length 546.I $\mu \mu$ and multiplying it by the constant 0.850944 . By this method the errors due to the character of the sodium source of light are eliminated and the measurements of one observer may be readily compared with those of another. 